\title{
ON QUOTIENTS OF TENSOR ALGEBRAS AND THEIR $C^{*}$-ENVELOPES
}

\author{
PAUL S. MUHLY ${ }^{1}$ AND BARUCH SOLEL ${ }^{2}$ \\ ${ }^{1}$ Department of Mathematics, University of Iowa, Iowa City, \\ IA 52242, USA (muhly@math.uiowa.edu) \\ ${ }^{2}$ Department of Mathematics, The Technion, 32000 Haifa, \\ Israel (mabaruch@techunix.technion.ac.il)
}

(Received 5 July 1998)

Abstract We identify the $C^{*}$-envelopes of certain quotients of tensor algebras over $C^{*}$-correspondences.

Keywords: tensor algebras; $C^{*}$-envelopes

AMS 1991 Mathematics subject classification: Primary 46H10;46H25; 46L99; 46M05

\section{Introduction}

Given an operator algebra $\mathcal{A}$, abstractly or concretely represented, one of the principal things to determine about it is its $C^{*}$-envelope, $C^{*}(\mathcal{A})$, in the sense of Arveson [1]. The $C^{*}$-envelope $C^{*}(\mathcal{A})$ codifies the matrix norm structure on $\mathcal{A}$ and provides a 'platform' from which to study the representation theory of the algebra. In [9], we studied the tensor algebra, $\mathcal{T}_{+}(E)$, over a $C^{*}$-correspondence $E$ and showed, under very general hypotheses on $E$, that the $C^{*}$-envelope of $\mathcal{T}_{+}(E)$ is the Cuntz-Pimsner algebra of $E, \mathcal{O}(E)$ [11]. Inspired by this success and the algebraic fact [6] that every finite-dimensional algebra over $\mathbb{C}$ is a quotient of a tensor algebra over the algebra modulo its radical, we have sought to identify the $C^{*}$-envelopes of quotients of tensor algebras of the form $\mathcal{T}_{+}(E)$. While our results are conditioned by a number of hypotheses, the class of quotients that are covered by our analysis covers a broad family of algebras and contains, in particular, many finite-dimensional algebras of current interest. It is hoped that our results will help provide a new mechanism for bringing the technology from finite-dimensional algebra, i.e. ring theory, to bear on problems in operator algebra.

We follow the notation and terminology from [9], but review the salient features that we need here. A $C^{*}$-correspondence over a $C^{*}$-algebra $A$ is a bimodule $E$ over $A$ such that as a right $A$-module, $E$ is a Hilbert $C^{*}$-module in the commonly accepted sense (see [7]), and, as a left $A$-module, the action of $A$ is given by bounded adjointable operators on $E$. That is, the left action is given by a $C^{*}$-homomorphism $\varphi: A \rightarrow \mathcal{L}(E)$. From a 
correspondence $E$ one can build another correspondence, $\mathcal{F}(E)$, called the (full) Fock space over $E$. By definition, $\mathcal{F}(E)$ is the direct sum $A \oplus E \oplus E^{\otimes 2} \oplus E^{\otimes 3} \oplus \cdots$. The powers $E^{\otimes n}$ are the internal tensor products of copies of $E$. Each is a correspondence over $A$. For example, at the level 2, the inner product on $E^{\otimes 2}$ is given by the formula

$$
\left\langle\xi_{1} \otimes \eta_{1}, \xi_{2} \otimes \eta_{2}\right\rangle=\left\langle\eta_{1}, \varphi\left(\left\langle\xi_{1}, \xi_{2}\right\rangle\right) \eta_{2}\right\rangle ;
$$

the right action of $A$ is given by the formula $\left(\xi_{1} \otimes \xi_{2}\right) a=\xi_{1} \otimes\left(\xi_{2} a\right)$; and the left action is given by the formula $\varphi_{2}(a)\left(\xi_{1} \otimes \xi_{2}\right)=\left(\varphi(a) \xi_{1}\right) \otimes \xi_{2}$. The higher powers are defined inductively. We write $\varphi_{\infty}$ for the left action of $A$ on $\mathcal{F}(E)$, i.e. $\varphi_{\infty}(a)=$ $\operatorname{diag}\left(\varphi_{0}(a), \varphi_{1}(a), \varphi_{2}(a), \ldots\right)$, where $\varphi_{0}(a)$ is left multiplication by $a$ on $A, \varphi_{1}=\varphi$, etc. For $\xi \in E$, the creation operator determined by $\xi, T_{\xi}$, is given by the formula $T_{\xi} \eta=\xi \otimes \eta$, $\eta \in \mathcal{F}(E)$. This operator belongs to $\mathcal{L}(\mathcal{F}(E))$, and its adjoint acts on tensors of the form $\eta \otimes \zeta, \eta \in E, \zeta \in \mathcal{F}(E)$, by the formula $T_{\xi}^{*} \eta \otimes \zeta=\varphi_{\infty}(\langle\xi, \eta\rangle) \zeta$. (Since such tensors span $\mathcal{F}(E), T_{\xi}^{*}$ is completely determined by this formula.) We extend the definition of $T_{\xi}$ to include elements $\xi \in E^{\otimes n}$ via the formula $T_{\xi}:=T_{\xi_{1}} T_{\xi_{2}} \cdots T_{\xi_{n}}$, if $\xi=\xi_{1} \otimes \xi_{2} \otimes \cdots \otimes \xi_{n}$. The tensor algebra of $E, \mathcal{T}_{+}(E)$, is defined to be the norm closed subalgebra of $\mathcal{L}(\mathcal{F}(E))$ generated by $\varphi_{\infty}(A)$ and the creation operators $T_{\xi}, \xi \in E$.

We write $\lambda$ for the gauge automorphism group of $\mathcal{T}_{+}(E)$. That is, $\lambda$ is given by the formula $\lambda_{t}\left(T_{\xi}\right)=\mathrm{e}^{\mathrm{i} t l} T_{\xi}$ for $\xi \in E^{\otimes l}$ and $\left.\lambda_{t}\right|_{\varphi_{\infty}(A)}=$ id. Also, we write $\Phi_{k}=\int \lambda_{t}(\cdot) \mathrm{e}^{-\mathrm{i} t k} \mathrm{~d} t$, so that $\Phi_{k}$ is the projection of $\mathcal{T}_{+}(E)$ onto $\left\{T_{\xi}: \xi \in E^{\otimes k}\right\}$.

In this note, we focus on ideals $\mathfrak{I}$ in $\mathcal{T}_{+}(E)$ that satisfy five hypotheses. First, we shall assume that

(H1) there is an $n$ (which will be fixed throughout this note) such that $J_{n} \subseteq \mathfrak{I} \subseteq J_{1}$, where $J_{k}=\overline{\operatorname{span}}\left\{T_{\xi}: \xi \in E^{\otimes l}, l \geqslant k\right\}$.

This hypothesis is motivated by considerations from ring theory. The ideals that appear in the representation of a finite-dimensional algebra as a quotient of a tensor algebra have this property. See [8] and our discussion in $\S 4$.

The second hypothesis is:

(H2) $\mathfrak{I}$ is $\lambda$-invariant.

This hypothesis is not automatically satisfied, even in the finite-dimensional setting. There are some situations in which it is automatic, however. For example, if $E$ is the correspondence which is $A$ as a right Hilbert module and where the left action is given by an automorphism of $A$, then the ideals of $\mathcal{T}_{+}(E)$ are $\lambda$-invariant when $A$ is simple and the Connes spectrum of the automorphism is the full circle [10]. On the other hand, we show in Example 4.2 that unexpected difficulties arise when it is not assumed. Hypothesis (H2) implies that $\mathfrak{I}$ is invariant under each of the mappings $\Phi_{k}$, and, hence, there are subbimodules $F_{k} \subseteq E^{\otimes k}$ such that

$$
\mathfrak{I}=\overline{\operatorname{span}}\left\{T_{\xi}: \xi \in F_{k}, k \geqslant 0\right\} .
$$

Since $J_{n} \subseteq \mathfrak{I} \subseteq J_{1}, F_{0}=\{0\}$ and $F_{k}=E^{\otimes k}$ for $k \geqslant n$. It is also evident from the fact that $\mathfrak{I}$ is an ideal that $F_{k} \otimes E \subseteq F_{k+1}$ and $E \otimes F_{k} \subseteq F_{k+1}$ for all $k$. In fact, whenever we 
choose $\left\{F_{k}\right\}$ satisfying these properties, then $\mathfrak{I}$, defined by (1.1), is a $\lambda$-invariant ideal such that $J_{n} \subseteq \mathfrak{I} \subseteq J_{1}$.

Our third hypothesis on the ideals we consider is

(H3) Each $F_{k}$ is complemented in $E^{\otimes k}$.

We write $\hat{F}_{k}$ for the projection in $\mathcal{L}(\mathcal{F}(E))$ whose range is $F_{k}$. Note that if $E$ is self-dual, then so is every $E^{\otimes k}$ and each sub-bimodule $F_{k}$ of $E^{\otimes k}$ is complemented. In particular, this hypothesis is satisfied when $E$ is associated to a finite quiver. (For the relation between quivers and $C^{*}$-correspondences (see $\S 4$ below and $[8,9,15]$ ).)

These three hypotheses guarantee that the quotient algebra $\mathcal{T}_{+}(E) / \mathfrak{I}$ is completely isometrically isomorphic to the compression of $\mathcal{T}_{+}(E)$ to the complement in $\mathcal{F}(E)$ of the space $\sum_{k=0}^{\infty} F_{k}$ (see Theorem 2.9). The projection, $P$, onto this space is $\sum_{k=0}^{\infty} Q_{k}-\hat{F}_{k}$, where $Q_{m}$ denotes the projection of $\mathcal{F}(E)$ onto $E^{\otimes m}$. This compression representation, in turn, depends on a distance formula that we take up in the next section. The fourth hypothesis that we make is that $\varphi(A)$ is contained in the compact operators on $E, K(E)$. This hypothesis is a common one for the theory of tensor algebras. It is satisfied, for example, if $E$ is a finitely generated projective module over $A$. In particular, it is satisfied for the algebras associated to finite quivers. The first four hypotheses, then, imply that the $C^{*}$-algebra on $P \mathcal{F}(E)$ generated by $P \mathcal{T}_{+}(E) P$ is all of $K(P \mathcal{F}(E)$ ). Finally, our fifth hypothesis, which is about annihilators of certain submodules of $P \mathcal{F}(E)$, is added to the first four to show that the $C^{*}$-envelope of $\mathcal{T}_{+}(E) / \mathfrak{I}$ is $K(P \mathcal{F}(E))$. While the fifth hypothesis may not be necessary for the $C^{*}$-envelope of $\mathcal{T}_{+}(E) / \mathfrak{I}$ to be $K(P \mathcal{F}(E)$ ), an example that we give in $\S 4$ shows that certain control of these annihilators is necessary.

\section{Distance formulae}

As just mentioned, the key to our analysis is to show that under our hypotheses (H1), $(\mathrm{H} 2)$ and $(\mathrm{H} 3)$, the quotient $\mathcal{T}_{+}(E) / \mathfrak{I}$ is completely isometrically isomorphic to $P \mathcal{T}_{+}(E) P$. This, in turn, requires being able to calculate the distance $d(T, I)$ from an operator $T \in \mathcal{T}_{+}(E)$ to $\mathfrak{I}$. We begin this calculation with the following lemma.

Lemma 2.1. Let $\mathfrak{I}$ be an ideal in $\mathcal{T}_{+}(E)$ satisfying hypotheses $(H 1)-(H 3)$, and, with the notation just established, set $\hat{E}_{k}=Q_{k}-\hat{F}_{k}$ and $P=\sum \hat{E}_{k}$. Then $I-P \in$ lat $\mathcal{T}_{+}(E)$ and

$$
\mathfrak{I}=\left\{T \in \mathcal{T}_{+}(E): P T P=0\right\}=\left\{T \in \mathcal{T}_{+}(E): P T=0\right\}
$$

Proof. If $\eta \in F_{k}$ and $\xi \in E^{\otimes l}$, then $T_{\xi} \eta=\xi \otimes \eta \in F_{k} \otimes E^{\otimes l} \subseteq F_{k+l}$. Hence, $T_{\xi} F_{k} \subseteq F_{k+l}$. Since $I-P=\sum \hat{F}_{k}, I-P \in$ lat $\mathcal{T}_{+}(E)$, and it follows that $P T P=P T$ for all $T \in \mathcal{T}_{+}(E)$. It is left to show that

$$
\mathfrak{I}=\left\{T \in \mathcal{T}_{+}(E): P T=0\right\} .
$$

Since for every $T \in \mathcal{T}_{+}(E), P T=\sum_{m=0}^{n-1} P \Phi_{m}(T)$ (because $P\left(E^{\otimes k}\right)=(0)$ for $k \geqslant n$, where $n$ is fixed in hypothesis (H1)), and since $\Phi_{m}(\mathfrak{I}) \subseteq \mathfrak{I}$ by hypothesis (H1), it suffices to show that, for $\xi \in E^{8 m}, T_{\xi} \in \mathfrak{I}$ if and only if $P T_{\xi}=0$. But $T_{\xi} \in \mathfrak{I}$ if and only if 
$\xi \in F_{m}$, and this implies that $P T_{\xi}=\sum \hat{E}_{k} T_{\xi}=0$ (as $F_{m} \otimes E^{\otimes l} \subseteq F_{m+l}$ ). On the other hand, $P T_{\xi}=0$ implies $\hat{E}_{m} T_{\xi} Q_{0}=0$, i.e. $\xi \in F_{m}$.

From now on, $P$ will always denote the projection $\sum \hat{E}_{k}$ of the lemma. Our goal, Theorem 2.9 , is to show that $\mathcal{T}_{+}(E) / \mathfrak{I}$ is completely isometrically isomorphic to $P \mathcal{T}_{+}(E) P$ acting on $P \mathcal{F}(E)$. For this purpose, the following distance formula is key.

Theorem 2.2. If $\mathfrak{I}$ is an ideal in $\mathcal{T}_{+}(E)$ satisfying the three hypotheses (H1)-(H3), then, for $T \in \mathcal{T}_{+}(E)$,

$$
\operatorname{dist}(T, \mathfrak{I})=\|P T P\| .
$$

Proof. The proof is broken into several steps and we formalize parts of it in two lemmas and a proposition. First, recall that $P_{k}$ is $\sum_{l=0}^{k} Q_{l}$ and write $P^{(k)}=P_{k}+\sum_{j=k+1}^{n-1} \hat{E}_{j}$. Then $\left\{P^{(k)}\right\}_{k=0}^{\infty}$ is an increasing sequence of projections in $\mathcal{L}(\mathcal{F}(E)), P^{(0)}=P$ and $P^{(k)}=P_{k}$ for $k \geqslant n-1$. Also note that $P^{(k+1)}=P^{(k)}+\hat{F}_{k+1}$. We shall assume that $\|P T P\|=q<1$ and fix $\varepsilon<1-q$. We shall construct, inductively, a sequence $\left\{T_{k}\right\}_{k=0}^{\infty}$ of operators in $T+\mathfrak{I}$ such that $T_{0}=T, P^{(k)} T_{j}=P^{(k)} T_{k}$ for $k \leqslant j$ and $\left\|P^{(k)} T_{k} P^{(k)}\right\| \leqslant q_{k}<1$ where $q_{k}=q+\varepsilon\left(\sum_{l=1}^{k} 2^{-l}\right)$. Since $T_{0}$ was set to be $T$, we describe the inductive step. Hence, we assume that we have already constructed $\left\{T_{j}\right\}_{j=0}^{m}$ as above $(m \geqslant 0)$.

First, we wish to construct $X=\hat{F}_{m+1} T_{m+1} P_{0}$. For this we consider the following operator matrix,

$$
S=\left(\begin{array}{cc}
P^{(m)} T_{m} P_{0} & P^{(m)} T_{m}\left(P^{(m+1)}-P_{0}\right) \\
X & \hat{F}_{m+1} T_{m}\left(P^{(m+1)}-P_{0}\right)
\end{array}\right),
$$

viewed as an operator from $P^{(m+1)} \mathcal{F}(E)=\left(P^{(m+1)}-P_{0}\right) \mathcal{F}(E) \oplus P_{0} \mathcal{F}(E)$ to $P^{(m+1)} \mathcal{F}(E)$, written as $P^{(m+1)} \mathcal{F}(E)=P^{(m)} \mathcal{F}(E) \oplus \hat{F}_{m+1} \mathcal{F}(E)$. Note that

$$
T_{m} F_{m+1} \subseteq \mathcal{T}_{+}(E) F_{m+1} \subseteq \sum_{j=m+1}^{\infty} F_{j} \subseteq\left(I-P^{(m)}\right) \mathcal{F}(E) .
$$

Hence, $P^{(m)} T_{m}\left(P^{(m+1)}-P_{0}\right)=P^{(m)} T_{m}\left(P^{(m)}-P_{0}\right)$. Thus, the first row of $S$ can be viewed as the operator $P^{(m)} T_{m} P^{(m)}$. By induction, its norm is dominated by $q_{m}$. Next, consider the second column of $S$. Here, note that $T_{m}\left(P^{(m+1)}-P_{0}\right)(\mathcal{F}(E)) \subseteq(I-$ $\left.P_{0}\right)(\mathcal{F}(E))$. Hence, the second column is $\left(P^{(m+1)}-P_{0}\right) T_{m}\left(P^{(m+1)}-P_{0}\right)$.

Lemma 2.3. $\left\|\left(P^{(m+1)}-P_{0}\right) T\left(P^{(m+1)}-P_{0}\right)\right\| \leqslant\left\|P^{(m)} T P^{(m)}\right\|$ for all $m, T \in \mathcal{T}_{+}(E)$.

Proof. If $m \geqslant n-1, P^{(m+1)}=P_{m+1}$ and $P^{(m)}=P_{m}$. Since we may identify $\left(P_{m+1}-\right.$ $\left.P_{0}\right)(\mathcal{F}(E))$ with $P_{m}(\mathcal{F}(E)) \otimes E$ and the identification carries $\left(P_{m+1}-P_{0}\right) T\left(P_{m+1}-P_{0}\right)$ into $P_{m} T P_{m} \otimes I_{E}$, the inequality in this case is clear. Now assume that $m<n-1$. We have

$$
P_{n-2}-\left(P^{(m)}-\hat{E}_{n-1}\right)=\sum_{j=m+1}^{n-2} \hat{F}_{j}
$$


and

$$
\left(P^{(m+1)}-P_{0}\right)\left(\left(\sum_{m+1}^{n-2} \oplus F_{j}\right) \otimes E\right) \subseteq\left(P^{(m+1)}-P_{0}\right)\left(\sum_{m+2}^{n-1} \oplus F_{j}\right)=\{0\} .
$$

Also, $I-P^{(m)}+\hat{E}_{n-1} \in$ lat $\mathcal{T}_{+}(E)$. Consequently, we have $\left(P^{(m)}-\hat{E}_{n-1}\right) T=\left(P^{(m)}-\right.$ $\left.\hat{E}_{n-1}\right) T\left(P^{(m)}-\hat{E}_{n-1}\right)$. We may then view $\left(P^{(m)}-\hat{E}_{n-1}\right) T$ as an operator on $P_{n-2}(\mathcal{F}(E))$ and $\left(P^{(m)}-\hat{E}_{n-1}\right) T \otimes I_{E}$ is an operator on $P_{n-1} \mathcal{F}(E)$ (identifying $P_{n-2}(\mathcal{F}(E)) \otimes E$ with $\left.P_{n-1} \mathcal{F}(E) \ominus P_{0} \mathcal{F}(E)\right)$. The computation above shows that

$$
\begin{aligned}
\left(P^{(m+1)}-P_{0}\right)\left[\left(P^{(m)}-\hat{E}_{n-1}\right) T\left(P^{(m)}-\hat{E}_{n-1}\right) \otimes I_{E}\right]\left(P^{(m+1)}-P_{0}\right) & \\
& =\left(P^{(m+1)}-P_{0}\right)\left[P_{n-2} T \otimes I_{E}\right]\left(P^{(m+1)}-P_{0}\right) .
\end{aligned}
$$

But the latter is $\left(P^{(m+1)}-P_{0}\right) T\left(P^{(m+1)}-P_{0}\right)$. Hence,

$$
\begin{aligned}
\left\|\left(P^{(m+1)}-P_{0}\right) T\left(P^{(m+1)}-P_{0}\right)\right\| & \leqslant\left\|\left(P^{(m)}-\hat{E}_{n-1}\right) T\left(P^{(m)}-\hat{E}_{n-1}\right)\right\| \\
& \leqslant\left\|P^{(m)} T P^{(m)}\right\| .
\end{aligned}
$$

Returning to our matrix (equation (2.1)), note that $P^{(m)} T_{m} P_{0}$ lies in $K\left(A, P^{(m)} \mathcal{F}(E)\right.$ ), $P^{(m)} T_{m}\left(P^{(m+1)}-P_{0}\right)$ lies in $K\left(\left(P^{(m+1)}-P_{0}\right) \mathcal{F}(E), P^{(m)} \mathcal{F}(E)\right)$ and $\hat{F}_{m+1} T_{m}\left(P^{(m+1)}-\right.$ $\left.P_{0}\right)$ lies in $K\left(\left(P^{(m+1)}-P_{0}\right) F_{m+1}\right)$. We can, therefore, apply the following lemma, which is a $C^{*}$-version of a well-known fact from operator theory [3, Lemma 9.1] (see also [4]) to find $X \in K\left(A, F_{m+1}\right)$ such that $\|S\| \leqslant q_{m+1}<1$ (recall that $q_{m}<q_{m+1}<1$ ).

Lemma 2.4. Let $\mathcal{A}$ be a $C^{*}$-algebra, let $p_{1}$ and $p_{1}^{\prime}$ be two projections in the multiplier algebra of $\mathcal{A}, M(\mathcal{A})$, let $p_{2}=1-p_{1}$, and let $p_{2}^{\prime}=1-p_{1}^{\prime}$. If $a \in p_{1}^{\prime} \mathcal{A} p_{1}, b \in p_{1}^{\prime} \mathcal{A} p_{2}$, and $c \in p_{2}^{\prime} \mathcal{A} p_{2}$ are such that $\max \{\|a+b\|,\|a+c\|\}=q_{1}<q_{2} \leqslant 1$, then there is an $x \in p_{2}^{\prime} \mathcal{A} p_{2}$ such that $\|a+b+c+x\| \leqslant q_{2}$.

Proof. It is helpful to think in terms of matrices, for then it is possible to follow the proof in [3, Lemma 9.1] almost word for word. We wish to find an $x \in p_{2}^{\prime} \mathcal{A} p_{2}$ that makes the norm of the matrix

$$
T=\left[\begin{array}{ll}
a & b \\
c & x
\end{array}\right]
$$

viewed as a transformation from $p_{1} \mathcal{A} \oplus p_{2} \mathcal{A}$ to $p_{1}^{\prime} \mathcal{A} \oplus p_{2}^{\prime} \mathcal{A}$, less than or equal to $q_{2}$ under the assumption that

$$
\max \left\{\left\|\left[\begin{array}{ll}
a & b
\end{array}\right]\right\|,\left\|\left[\begin{array}{l}
a \\
c
\end{array}\right]\right\|\right\}=q_{1}<q_{2} \leqslant 1 .
$$

(The strict inequality; $q_{1}<1$, is essential for the general $C^{*}$-algebra setting. For operators on Hilbert space, one can get away with $q_{1}=1$.) From the inequality

$$
\left\|\left[\begin{array}{l}
a \\
c
\end{array}\right]\right\| \leqslant q_{1}<q_{2} \leqslant 1
$$


we conclude that

$$
1 \geqslant q_{2}^{2} \cdot 1>q_{1}^{2} \cdot 1 \geqslant\left[\begin{array}{l}
a \\
c
\end{array}\right]^{*}\left[\begin{array}{l}
a \\
c
\end{array}\right]=a^{*} a+c^{*} c
$$

Thus, $q_{2}^{2}-a^{*} a \geqslant c^{*} c$ and $q_{2}^{2}-a^{*} a$ is invertible. Therefore, so is $1-a_{1}^{*} a_{1}$, where $a_{1}=q_{2} a$ (because $\left.q_{2}^{2}-a^{*} a=q_{2}^{2}\left(1-a_{1}^{*} a_{1}\right)\right)$. Since $q_{2}^{2}\left(1-a_{1}^{*} a_{1}\right) \geqslant c^{*} c, q_{2}^{2} \geqslant\left(1-a_{1}^{*} a_{1}\right)^{-1 / 2} c^{*} c(1-$ $\left.a_{1}^{*} a_{1}\right)^{-1 / 2}$. Consequently, if we set $l=c\left(1-a_{1}^{*} a_{1}\right)^{-1 / 2}$, then this inequality shows that $\|l\| \leqslant q_{2}$. Similarly, we conclude that $b b^{*} \leqslant q_{2}^{2}-a a^{*}$, with $q_{2}^{2}-a a^{*}$ invertible. Now we set $k:=q_{2}^{-1} b^{*}\left(1-a_{1} a_{1}^{*}\right)^{-1 / 2}$. Calculating as before, we see that $\|k\| \leqslant 1$. Then, for $x=-l a_{1}^{*} k^{*}$, we see that

$$
T=\left[\begin{array}{ll}
a & b \\
c & x
\end{array}\right]=\left[\begin{array}{cc}
q_{2} 1 & 0 \\
0 & l
\end{array}\right]\left[\begin{array}{cc}
a_{1} & \left(1-a_{1} a_{1}^{*}\right)^{1 / 2} \\
\left(1-a_{1}^{*} a_{1}\right)^{1 / 2} & -a_{1}^{*}
\end{array}\right]\left[\begin{array}{cc}
1 & 0 \\
0 & k^{*}
\end{array}\right] .
$$

The first matrix in the product has norm at most $q_{2}$. The inner matrix is unitary, as a straightforward calculation shows. Finally, the third matrix has norm at most 1 . Thus, $\|T\| \leqslant q_{2}$, as required.

In our case the algebra $\mathcal{A}$ is $K\left(P^{(m+1)} \mathcal{F}(E)\right), p_{1}=P^{(m+1)}-P_{0}$, and $p_{1}^{\prime}=P^{(m)}$. Since the $X$ we are seeking lies in $K\left(A, F_{m+1}\right)$, it is associated with a unique vector $\eta \in F_{m+1}$. Then $T_{\eta} \in \mathfrak{I}$ (by the definition of $F_{m+1}$ ). There is a vector $g \in E^{\otimes(m+1)}$ such that $\Phi_{m+1}\left(T_{m}\right)=T_{g}$. Set

$$
T_{m+1}=T_{m}-T_{\hat{F}_{m+1} g}+T_{\eta}
$$

Then

$$
T_{m+1} \in T_{m}+\mathfrak{I}=T+\mathfrak{I} .
$$

Since $P^{(k)} T_{\xi}=0$ for all $\xi \in F_{m+1}$ and $k \leqslant m, P^{(k)} T_{m+1}=P^{(k)} T_{m}=P^{(k)} T_{k}$ (by the induction hypothesis).

It is left to show that $\left\|P^{(m+1)} T_{m+1} P^{(m+1)}\right\| \leqslant q_{m+1}$. For this we need to show that $S=P^{(m+1)} T_{m+1} P^{(m+1)}$. It is easy to check that for every $\xi \in F_{m+1}$ we have $P^{(m)} T_{\xi} P_{0}=$ $P^{(m)} T_{\xi}\left(P^{(m+1)}-P_{0}\right)=\hat{F}_{m+1} T_{\xi}\left(P^{(m+1)}-P_{0}\right)=0$. Hence, three entries of $S$ agree with those of $P^{(m+1)} T_{m+1} P^{(m+1)}$. Finally, since it is easy to see that $\hat{F}_{m+1} T_{m} P_{0}=$ $\hat{F}_{m+1} T_{\hat{F}_{m+1} g} P_{0}$, the desired equality is proved.

We summarize formally what we have done so far in the following proposition.

Proposition 2.5. Assume $T \in \mathcal{T}_{+}(E)$ satisfies $\|P T P\|<1$ and write $q=\|P T P\|$. Fix $0<\varepsilon<1-q$ and set $q_{k}=q+\varepsilon\left(\sum_{l=1}^{k} 2^{-l}\right)$. Then we can construct a sequence of operators $\left\{T_{k}: 0 \leqslant k\right\}$ in $T+\mathfrak{I}$ satisfying:

(1) $T_{0}=T$;

(2) for $k \leqslant j, P^{(k)} T_{j}=P^{(k)} T_{k}$; and

(3) for all $k,\left\|P^{(k)} T_{k} P^{(k)}\right\| \leqslant q_{k}$. 
To complete the proof that $\operatorname{dist}(T, \mathfrak{I})<1$, we introduce the following notation. For $m \geqslant 0$, we write $k_{m}$ for the $m$ th Fejer kernel and for $R \in \mathcal{T}_{+}(E)$, we set

$$
k_{m} * R=\int k_{m}(t) \lambda_{t}(R) \mathrm{d} t
$$

Then

$$
k_{m} * R=\sum_{j=0}^{m}\left(1-\frac{j}{m}\right) \Phi_{j}(R) \in \mathcal{T}_{+}(E) .
$$

With $q$ and $\varepsilon$ as in the proposition, write $q^{\prime}=q+\varepsilon(<1)$ and fix $m>n$ such that $\left(n^{2} / m\right)<1-q^{\prime}$. Set

$$
C:=\sum_{j=0}^{n} \Phi_{j}\left(T_{m}\right)+\sum_{j=n+1}^{m}\left(1-\frac{j}{m}\right) \Phi_{j}\left(T_{m}\right)
$$

Then we have

$$
C-\sum_{j=0}^{n} \Phi_{j}\left(T_{m}\right) \in J_{n+1} \subseteq \mathfrak{I}
$$

and

$$
T_{m}-\sum_{j=0}^{n} \Phi_{j}\left(T_{m}\right) \in J_{n+1} \subseteq \mathfrak{I} .
$$

Consequently, $C-T_{m} \in \mathfrak{I}$ and $C \in T_{m}+\mathfrak{I}=T+\mathfrak{I}$. Since $\left\|P_{n} T_{m} P_{n}\right\|=\left\|P^{(n)} T_{m} P^{(n)}\right\|=$ $\left\|P^{(n)} T_{n} P^{(n)}\right\| \leqslant q_{n}$, it easily follows that $\left\|\Phi_{j}\left(T_{m}\right)\right\| \leqslant q_{n}$ for all $0<j \leqslant n$. (This follows from the inequality $\left\|\Phi_{j}\left(T_{m}\right)\right\|=\left\|Q_{j} \Phi_{j}\left(T_{m}\right) Q_{0}\right\|=\left\|Q_{j} T_{m} Q_{0}\right\| \leqslant\left\|P_{n} T_{m} P_{n}\right\|$.) Hence,

$$
\begin{aligned}
\left\|C-k_{m} * T_{m}\right\| & =\left\|\sum_{j=0}^{n} \frac{j}{m} \Phi_{j}\left(T_{m}\right)\right\| \leqslant \sum_{j=0}^{n} \frac{j}{m} q_{n} \\
& \leqslant \frac{n^{2}}{m} q_{n}<\left(1-q^{\prime}\right) q_{n}<1-q^{\prime} .
\end{aligned}
$$

Recall that for every $j>m, P_{m} T_{j}=P_{m} T_{m}$ by item (2) of Proposition 2.5. Hence, $\Phi_{k}\left(T_{m}\right)=\Phi_{k}\left(T_{j}\right)$ for all $0 \leqslant k \leqslant m$, and so $k_{m} * T_{m}=k_{m} * T_{j}$ for all $j \geqslant m$. We find, then, that

$$
P_{j}\left(k_{m} * T_{m}\right)=P_{j}\left(k_{m} * T_{j}\right) P_{j}=k_{m} *\left(P_{j} T_{j} P_{j}\right)
$$

and

$$
\left\|P_{j}\left(k_{m} * T_{m}\right)\right\| \leqslant\left\|k_{m}\right\|_{1}\left\|P_{j} T_{j} P_{j}\right\| \leqslant q^{\prime} .
$$

It follows that $\left\|k_{m} * T_{m}\right\| \leqslant q^{\prime}$ and

$$
\|C\| \leqslant\left\|C-k_{m} * T_{m}\right\|+\left\|k_{m} * T_{m}\right\|<1-q^{\prime}+q^{\prime}=1
$$


Since $C \in T+\mathfrak{I}$, this proves that $\operatorname{dist}(T, \mathfrak{I})<1$, and, hence, that $\operatorname{dist}(T, \mathfrak{I})<1$. Thus, $\operatorname{dist}(T, \mathfrak{I}) \leqslant\|P T P\|$. The opposite inequality follows from the fact that for every $B \in \mathfrak{I}$,

$$
\|P T P\|=\|P(T+B) P\| \leqslant\|T+B\|,
$$

and so, by taking the infimum,

$$
\|P T P\| \leqslant \operatorname{dist}(T, \mathfrak{I}) .
$$

This completes the proof of the theorem.

Theorem 2.2 shows that, under hypotheses (H1)-(H3) made on $\mathfrak{I}$, the map $T \mapsto P T P$ induces an isometric isomorphism between $\mathcal{T}_{+}(E) / \mathfrak{I}$ and $P \mathcal{T}_{+}(E) P$. We wish to show that this map is, in fact, a complete isometry. For this it is enough to show that, for $n \in \mathbb{N}, \mathcal{T}_{+}\left(M_{n}(E)\right) \cong M_{n}\left(\mathcal{T}_{+}(E)\right)$ and that the ideal $M_{n}(\mathfrak{I})$ in $M_{n}\left(\mathcal{T}_{+}(E)\right)$ satisfies hypotheses (H1)-(H3).

To this end, we write $M_{n}(E), 1 \leqslant n \leqslant \infty$, for the $C^{*}$-module $M_{n}(\mathbb{C}) \otimes_{\mathrm{e}} E$, where $\otimes_{\mathrm{e}}$ is the exterior tensor product of the $M_{n}(\mathbb{C})$-module $M_{n}(\mathbb{C})$ and the $A$-module $E$, and where $M_{\infty}(\mathbb{C})$ is interpreted as $K\left(\ell_{2}\right)$. Hence, $M_{n}(E)$ is a right Hilbert $C^{*}$-module over $M_{n}(A)$. One can easily check that the inner product is

$$
\left\langle\left(e_{i j}\right),\left(e_{i j}^{\prime}\right)\right\rangle=\left(\sum_{k}\left\langle e_{k i}, e_{k j}^{\prime}\right\rangle_{A}\right) \in M_{n}(A),
$$

and that right action of $M_{n}(A)$ is given by the formula

$$
\left(e_{i j}\right)\left(a_{i j}\right)=\left(\sum_{k} e_{i k} a_{k j}\right) .
$$

The module $M_{n}(E)$ also comes equipped with a left action of $M_{n}(A)$ given by the equation:

$$
\varphi^{(n)}\left(\left(a_{i j}\right)\right)\left(e_{i j}\right)=\left(\sum_{k} \varphi\left(a_{i k}\right) e_{k j}\right)
$$

This makes $M_{n}(E)$ into a $C^{*}$-correspondence over $M_{n}(A)$. Consequently, we may form $\mathcal{F}\left(M_{n}(E)\right)$ and $\mathcal{T}_{+}\left(M_{n}(E)\right)$.

Lemma 2.6. For $C^{*}$-correspondences $E$ and $F$ (over $A$ ):

$$
M_{n}(E) \otimes_{M_{n}(A)} M_{n}(F) \simeq M_{n}\left(E \otimes_{A} F\right) .
$$

Proof. This is a consequence of the following well-known more-general fact. Suppose $E$ is a Hilbert $A$ module, that $F$ is a Hilbert $A^{\prime}$ module, and that $\varphi$ is a *-homomorphism from $A$ to $\mathcal{L}(F)$. Then, for any $C^{*}$-algebra $B$, we have $\left(B \otimes_{\mathrm{e}} E\right) \otimes_{\text {id } \otimes_{\varphi}}\left(B \otimes_{\mathrm{e}} F\right) \simeq$ $B \otimes_{\mathrm{e}}\left(E \otimes_{\varphi} F\right)$, where $\otimes_{\mathrm{e}}$ denotes the external tensor product as above and $\otimes_{\mathrm{id}} \otimes_{\varphi}$ and $\otimes_{\varphi}$ denote internal tensor products. (This follows easily from the development of the external tensor product given in [7, pp. 34-39].) In the present setting, $A=A^{\prime}$ and $B=M_{n}(\mathbb{C})$. 
So $\left(B \otimes_{\mathrm{e}} E\right) \otimes_{\mathrm{id} \otimes \varphi}\left(B \otimes_{\mathrm{e}} F\right)=M_{n}(E) \otimes_{M_{n}(A)} M_{n}(F)$ and $B \otimes_{\mathrm{e}}\left(E \otimes_{\varphi} F\right)=M_{n}\left(E \otimes_{A} F\right)$. It is also useful to note, and easy to check, that the formula

$$
W\left(\left(e_{i j}\right) \otimes\left(f_{i j}\right)\right)=\left(\sum_{k} e_{i k} \otimes f_{k j}\right)
$$

$\left(e_{i j}\right) \in M_{n}(E),\left(f_{i j}\right) \in M_{n}(F)$, defines the desired isomorphism. We omit further details, which are quite straightforward.

Proposition 2.7. The isomorphism of Lemma 2.6 extends to a $C^{*}$-correspondence isomorphism

$$
V: \mathcal{F}\left(M_{n}(E)\right) \rightarrow M_{n}(\mathcal{F}(E))
$$

Further, via conjugation, see see that $V$ induces a $C^{*}$-isomorphism $\Psi: \mathcal{L}\left(\mathcal{F}\left(M_{n}(E)\right)\right) \rightarrow$ $M_{n}(\mathcal{L}(\mathcal{F}(E))), \Psi(T)=V T V^{-1}$ for $T \in \mathcal{L}\left(\mathcal{F}\left(M_{n}(E)\right)\right)$, such that $\Psi\left(\mathcal{T}_{+}\left(M_{n}(E)\right)\right)=$ $M_{n}\left(\mathcal{T}_{+}(E)\right)$ and such that if $\lambda_{t}^{(n)}$ denotes the gauge action on $\mathcal{T}_{+}\left(M_{n}(E)\right)$ and if $\left(T_{i j}\right) \in$ $M_{n}\left(\mathcal{T}_{+}(E)\right)$, then

$$
\Psi \circ \lambda_{t}^{(n)} \circ \Psi^{-1}\left(\left(T_{i j}\right)\right)=\left(\lambda_{t}\left(T_{i j}\right)\right)
$$

Proof. Let $\xi=\left(\xi_{i j}\right) \in M_{n}(E)$ and let $\eta \in M_{n}(\mathcal{F}(E))$ be of the form $\eta=\left(\eta_{i j}\right)$, where

$$
\eta_{i j}= \begin{cases}0, & \text { if }(i, j) \neq\left(i_{0}, j_{0}\right), \\ e_{1} \otimes e_{2} \otimes \cdots \otimes e_{k}, & \text { if }(i, j)=\left(i_{0}, j_{0}\right)\end{cases}
$$

(such elements span $M_{n}(\mathcal{F}(E))$ ). Then

$$
V^{-1} \eta=e_{1} E_{i_{0} i_{0}} \otimes e_{2} E_{i_{0} i_{0}} \otimes \cdots \otimes e_{k} E_{i_{0} j_{0}}
$$

where $e E_{l r}$ denotes the element of $M_{n}(E)$ whose $l, r$ entry is $e$ and whose other entries are zeros, and

$$
T_{\xi} V^{-1} \eta=\xi \otimes V^{-1} \eta=\sum_{l} \xi_{l i_{0}} E_{l i_{0}} \otimes e_{1} E_{i_{0} i_{0}} \otimes \cdots \otimes e_{k} E_{i_{0} j_{0}}
$$

Hence,

$$
V T_{\xi} V^{-1} \eta=\sum_{l} \xi_{l i_{0}} \otimes e_{1} \otimes \cdots \otimes e_{k} E_{l j_{0}}
$$

On the other hand,

$$
\left(T_{\xi_{i j}}\right) \eta=\left(T_{\xi_{i j}}\right)\left(e_{1} \otimes \cdots \otimes e_{k}\right) E_{i_{0} j_{0}}=\sum \xi_{i i_{0}} \otimes e_{1} \otimes \cdots \otimes e_{k} E_{i j_{0}}=V T_{\xi} V^{-1} \eta
$$

Hence, $\Psi\left(T_{\xi}\right)=\left(T_{\xi_{i j}}\right)$, where $\xi=\left(\xi_{i j}\right)$. The fact that $\Psi$ carries $\mathcal{T}_{+}\left(M_{n}(E)\right)$ onto $M_{n}\left(\mathcal{T}_{+}(E)\right)$ is now clear. The fact that $\Psi$ intertwines the gauge actions is also clear from the formula

$$
\Psi\left(T_{\xi}\right)=\left(T_{\xi_{i j}}\right)
$$


Corollary 2.8. If $\mathfrak{I} \subseteq \mathcal{T}_{+}(E)$ is a $\lambda$-invariant ideal, then $M_{n}(\mathfrak{I}) \subseteq M_{n}\left(\mathcal{T}_{+}(E)\right)$ is mapped by $\Psi^{-1}$ onto a $\lambda^{(n)}$-invariant ideal in $\mathcal{T}_{+}\left(M_{n}(E)\right)$.

Suppose that $\mathfrak{I}$ satisfies hypotheses (H1)-(H3). Let $F_{k}$ be the associated submodules $\left(F_{k} \subseteq E^{\otimes k}\right)$ with corresponding projections $\hat{F}_{k} \in \mathcal{L}\left(E^{\otimes k}\right)$. Then, for $\xi \in M_{n}(E)^{\otimes k}=$ $V^{-1}\left(M_{n}\left(E^{\otimes k}\right)\right)$,

$$
\begin{aligned}
T_{\xi} \in \Psi^{-1}\left(M_{n}(\mathfrak{I})\right) & \Longleftrightarrow V \xi \in M_{n}\left(F_{k}\right) \\
& \Longleftrightarrow \xi \in V^{-1}\left(M_{n}\left(F_{k}\right)\right)
\end{aligned}
$$

If $E_{k}$ is the orthogonal complement of $F_{k}$ (in $E^{\otimes k}$ ), then $M_{n}\left(E_{k}\right)$ is the orthogonal complement of $M_{n}\left(F_{k}\right)$ and $V^{-1}\left(M_{n}\left(E_{k}\right)\right)$ is the orthogonal complement of $V^{-1}\left(M_{n}\left(F_{k}\right)\right)$ in $M_{n}(E)^{\otimes k}$. Hence, $\Psi^{-1}\left(M_{n}(\mathfrak{I})\right)$ satisfies hypotheses (H1)-(H3), and so Theorem 2.2 allows us to conclude that for $\left(T_{i j}\right) \in M_{n}\left(\mathcal{T}_{+}(E)\right)$,

$$
\operatorname{dist}\left(\left(T_{i j}\right), M_{n}(\mathfrak{I})\right)=\left\|\tilde{P}\left(T_{i j}\right) \tilde{P}\right\|,
$$

where $\tilde{P} \in \mathcal{L}\left(M_{n}(\mathcal{F}(E))\right)$ is the projection corresponding to $M_{n}(\mathfrak{I})$. However, $\tilde{P}=$ $\operatorname{diag}(P, P, \ldots, P)$. Hence

$$
\operatorname{dist}\left(\left(T_{i j}\right), M_{n}(\mathfrak{I})\right)=\left\|\left(P T_{i j} P\right)\right\|
$$

Since

$$
\begin{aligned}
\left\|\left(T_{i j}+\mathfrak{I}\right)\right\|_{M_{n}\left(\mathcal{T}_{+}(E) / \mathfrak{I}\right)} & =\left\|\left(T_{i j}\right)+M_{n}(\mathfrak{I})\right\|_{M_{n}\left(\mathcal{T}_{+}(E)\right) / M_{n}(\mathfrak{I})} \\
& =\operatorname{dist}\left(\left(T_{i j}\right), M_{n}(\mathfrak{I})\right),
\end{aligned}
$$

we conclude that the following theorem holds.

Theorem 2.9. Let $\mathfrak{I} \subseteq \mathcal{T}_{+}(E)$ be an ideal satisfying hypotheses (H1)-(H3). Then the $\operatorname{map}$

$$
\mathcal{T}_{+}(E) \ni T \longmapsto P T P
$$

(with $P=\sum_{k} Q_{k}-\hat{F}_{k}$ ) induces a completely isometric isomorphism of $\mathcal{T}_{+}(E) / \mathfrak{I}$ onto $P \mathcal{T}_{+}(E) P$.

\section{3. $C^{*}$-envelopes}

The fourth hypothesis we place on our correspondence $E$ is

(H4) the range of $\varphi$ is contained in $K(E)$.

As we mentioned at the outset, this condition is common in the theory. It is satisfied when $E$ is finitely generated and projective.

Proposition 3.1. With the notation already established, under hypothesis (H4), the algebra $P \mathcal{T}_{+}(E) P$ is contained in $K(P \mathcal{F}(E))$ and generates $K(P \mathcal{F}(E))$ as a $C^{*}$-algebra. 
Proof. Since $\varphi(A) \subseteq K(E)$, we also have $K\left(E^{\otimes k}\right) \otimes I_{E} \subseteq K\left(E^{\otimes(k+1)}\right)$ (see [11]). For $\xi \in E$ and $k \geqslant 1,\left\|T_{\xi}-T_{\xi} \varphi_{\infty}\left(e_{\alpha}\right)\right\|=\left\|T_{\xi-\xi e_{\alpha}}\right\| \leqslant\left\|\xi-\xi e_{\alpha}\right\| \rightarrow 0$ when $\left\{e_{\alpha}\right\}$ is a contractive approximate identity in $A$. Since the restriction of $T_{\xi} \varphi_{\infty}\left(e_{\alpha}\right)$ to $E^{\otimes k}$ lies in $K\left(E^{\otimes k}, E^{\otimes(k+1)}\right)\left(\right.$ as $\left.\left.\varphi_{\infty}\left(e_{\alpha}\right)\right|_{E^{\otimes k}}=\varphi_{k}\left(e_{\alpha}\right) \in K\left(E^{\otimes k}\right)\right),\left.T_{\xi}\right|_{E^{\otimes k}} \in K\left(E^{\otimes k}, E^{\otimes(k+1)}\right)$. It then follows that $P \mathcal{T}_{+}(E) P \subseteq K(P(\mathcal{F}(E)))$. Now write $\mathcal{C}$ for the $C^{*}$-algebra generated by $P \mathcal{T}_{+}(E) P$ in $K(P \mathcal{F}(E))$. We shall show that for $0 \leqslant i \leqslant j \leqslant n-1, \mathcal{C}$ contains $K\left(E_{i}, E_{j}\right)$. As $P=\sum \hat{E}_{i}$ this will complete the proof. In fact, we show, by induction on $k$, that for every $0 \leqslant m \leqslant n-1$ and $0 \leqslant k \leqslant n-1, K\left(E_{m-k}, E_{n-k-1}\right) \subseteq \mathcal{C}$. Suppose $k=0$ and note that $\mathcal{C}$ contains $P T_{\xi} P T_{\eta}^{*} P$ for every $\xi \in E_{n-1}, \eta \in E_{m}$. But $P T_{\xi} P T_{\eta}^{*} P=\hat{E}_{n-1} T_{\xi} \hat{E}_{0} T_{\eta}^{*} \hat{E}_{m}=\hat{E}_{n-1} T_{\xi} T_{\eta}^{*} \hat{E}_{m}$. Varying $\xi$ and $\eta$ gives $K\left(E_{m}, E_{n-1}\right) \subseteq \mathcal{C}$. For the inductive step take $\xi \in E_{n-k-1}, \eta \in E_{m-k}$. Then $\mathcal{C}$ contains $P T_{\xi} P T_{\eta}^{*} P=$ $\sum_{j=0}^{k} E_{n-j-1} T_{\xi} T_{\eta}^{*} E_{m-j}$. Using the induction hypothesis,

$$
\sum_{j=0}^{k-1} E_{n-j-1} T_{\xi} T_{\eta}^{*} E_{m-j} \in \sum_{j=0}^{k-1} K\left(E_{m-j}, E_{n-j-1}\right) \subseteq \mathcal{C}
$$

Hence, $E_{n-k-1} T_{\xi} T_{\eta}^{*} E_{m-k} \in \mathcal{C}$ for all such $\xi, \eta$. Hence, $K\left(E_{m-k}, E_{n-k-1}\right) \subseteq \mathcal{C}$.

While hypothesis $(\mathrm{H} 4)$ guarantees that the $C^{*}$-algebra generated by $P \mathcal{T}_{+}(E) P$ is $K(P \mathcal{F}(E))$, in general, this is not its $C^{*}$-envelope. The following theorem presents a general sufficient condition for the $C^{*}$-envelope of $P \mathcal{T}_{+}(E) P$ to be $K(P \mathcal{F}(E)$ ). Before we state the theorem we need some notation. For a $C^{*}$-correspondence $F$ (over $A$ ) we write

$$
\operatorname{ann}(F)=\{a \in A: F a=0\}
$$

and

$$
k(F)=\{a \in A: \varphi(a) F=0\}
$$

With $P=\sum_{k=0}^{n-1} \hat{E}_{k}$ as above we write

$$
k_{m}=k\left(E_{m}\right)
$$

Our fifth hypothesis, then, is

(H5) The intersection

$$
\operatorname{ann}\left(E_{n-1}\right) \cap \operatorname{ann}\left(E_{n-2} k_{1}\right) \cap \operatorname{ann}\left(E_{n-3} k_{1} k_{2}\right) \cap \cdots \cap \operatorname{ann}\left(k_{1} \cdots k_{n-1}\right)
$$

vanishes.

Theorem 3.2. If $\mathfrak{I}$ is an ideal in $\mathcal{T}_{+}(E)$ satisfying hypotheses $(H 1)-(H 5)$, then the $C^{*}$-envelope of $\mathcal{T}_{+}(E) / \mathfrak{I}$ is $K(P \mathcal{F}(E))$. 
Proof. Recall that if $\mathcal{A}$ is an operator algebra generating a $C^{*}$-algebra $\mathcal{B}$ (as a $C^{*}$ algebra), then an ideal $\mathcal{I}$ in $\mathcal{B}$ is called a boundary ideal for $\mathcal{A}$ in case the quotient map $q: \mathcal{B} \rightarrow \mathcal{B} / \mathcal{I}$ is completely isometric when restricted to $\mathcal{A}$. The $C^{*}$-algebra $\mathcal{B}$ is the $C^{*}$ envelope of $\mathcal{A}$ precisely when only the zero ideal in $\mathcal{B}$ is a boundary ideal (see [1]). Thus, to show that $K(P \mathcal{F}(E))$ is the $C^{*}$-envelope of $\mathcal{T}_{+}(E) / \mathfrak{I}$, which is completely isometrically isomorphic to $P \mathcal{T}_{+}(E) P$, it suffices to show that under hypotheses (H4) and (H5), every non-zero ideal of $K(P \mathcal{F}(E))$ intersects $P \mathcal{T}_{+}(E) P$ non-trivially.

Note first that every ideal of $K(P \mathcal{F}(E))$ is of the form $K\left(P \mathcal{F}(E) \mathfrak{I}_{0}\right)$, where $\mathfrak{I}_{0}$ is an ideal in $A[14]$. So we fix a non-zero ideal $\mathfrak{I}_{0}$ in $A$ and prove

$$
K\left(P \mathcal{F}(E) \mathfrak{I}_{0}\right) \cap P \mathcal{T}_{+}(E) P \neq\{0\} .
$$

Since $\mathfrak{I}_{0} \neq 0$, there is an $m, 1 \leqslant m \leqslant n$, such that

$$
\mathfrak{I}_{0} \nsubseteq \operatorname{ann}\left(E_{n-m} k_{1} \cdots k_{m-1}\right)
$$

(for $m=1, k_{1} \cdots k_{m-1}$ is replaced by $A$ ). Hence, there is an $a \in \mathfrak{I}_{0}$ such that

$$
E_{n-m} k_{1} \cdots k_{m-1} a \neq 0 \text {. }
$$

Fix $\xi \in E_{n-m}$ and $b \in k_{1} \cdots k_{m-1}$ such that $\xi b a \neq 0$. Then

$$
\langle\xi b a, \xi b a\rangle=a^{*} b^{*}\langle\xi, \xi\rangle b a \in k_{1} \cdots k_{m-1} .
$$

Thus, $\varphi_{j}(\langle\xi b a, \xi b a\rangle) \eta=0$ for all $\eta \in E_{j}, 1 \leqslant j \leqslant m-1$, and, therefore,

$$
\xi b a \otimes \eta=0, \quad \text { for all } \eta \in E_{j}, 1 \leqslant j \leqslant m-1,
$$

i.e.

$$
\left.T_{\xi b a}\right|_{E_{1}+\cdots+E_{m-1}}=0 .
$$

We have

$$
P T_{\xi b a} P=\sum_{j=0}^{m-1} \hat{E}_{j+n-m} T_{\xi b a} \hat{E}_{j}=\hat{E}_{n-m} T_{\xi b a} \hat{E}_{0}=\hat{E}_{n-m} T_{\xi b a} .
$$

Since $\xi b a \neq 0$ and $T_{\xi b a} \notin \mathfrak{I}$ because $\xi b a \in E_{n-m}, P T_{\xi b a} P \neq 0$. Also, $\left.T_{\xi b}\right|_{A} \in K\left(A, E_{n-m}\right)$ and

$$
P T_{\xi b a} P=\hat{E}_{n-m}\left(\left.T_{\xi b}\right|_{A}\right)\left(\left.\varphi_{\infty}(a)\right|_{A}\right) .
$$

We now check that, whenever $a^{\prime} \in \mathfrak{I}_{0}$,

$$
\hat{E}_{n-m} K\left(A, E_{n-m}\right)\left(\left.\varphi_{\infty}\left(a^{\prime}\right)\right|_{A}\right) \subseteq K\left(\mathcal{I}_{0}, E_{n-m} \mathfrak{I}_{0}\right) .
$$

For this take $\theta \otimes b^{*} \in K\left(A, E_{n-m}\right)$ (since such elements span a dense subset of $K\left(A, E_{n-m}\right.$ ) it is enough to consider these) and compute, for $c \in A$,

$$
\begin{aligned}
\hat{E}_{n-m}\left(\theta \otimes b^{*}\right)\left(\left.\varphi_{\infty}\left(a^{\prime}\right)\right|_{A}\right)(c) & =\hat{E}_{n-m}\left(\theta \otimes b^{*}\right)\left(a^{\prime} c\right) \\
& =\hat{E}_{n-m} \theta b^{*} a^{\prime} c \\
& =\lim _{\alpha} \hat{E}_{n-m} \theta b^{*} e_{\alpha} a^{\prime} c
\end{aligned}
$$


where $\left\{e_{\alpha}\right\}$ is a contractive approximate identity in $\mathfrak{I}_{0}$. Hence,

$$
\hat{E}_{n-m}\left(\theta \otimes b^{*}\right)\left(\left.\varphi_{\infty}\left(a^{\prime}\right)\right|_{A}\right)=\lim _{\alpha}\left(\hat{E}_{n-m} \theta b^{*} e_{\alpha}\right) \otimes\left(a^{\prime *}\right)^{*}
$$

lies in $K\left(\mathfrak{I}_{0}, E_{n-m} \mathfrak{I}_{0}\right)$. This proves that $P T_{\xi b a^{\prime}} P$ is a non-zero element in $P \mathcal{T}_{+}(E) P \cap$ $K\left(P \mathcal{F}(E) \mathfrak{I}_{0}\right)$ and completes the proof.

Corollary 3.3. If $E$ is a $C^{*}$-correspondence satisfying hypothesis (H4) and if $E$ is full (i.e. if $\overline{\langle E, E\rangle}=A$ ) and essential as a left $A$-module (i.e. if $\varphi(A) E=E$ ), then for every $n>1$,

$$
C^{*}\left(\mathcal{T}_{+}(E) / J_{n}\right) \simeq K\left(\left(1-P_{n}\right) \mathcal{F}(E)\right)
$$

Proof. Here, $\mathfrak{I}=J_{n}$ satisfies hypotheses (H1)-(H3), trivially. Hypothesis (H4) is part of the hypotheses of the corollary. We shall show that fullness and essentiality imply hypothesis (H5). Note that $P=P_{n}$ and $E_{j}=E^{\otimes j}$ for $0 \leqslant j \leqslant n-1$. We will show, by induction, that each $E^{\otimes n}$ is full and essential. This will imply that $\operatorname{ann}\left(E^{\otimes n}\right)=0$ for all $n \geqslant 1$. (In fact, if $a \in \operatorname{ann}\left(E^{\otimes n}\right)$, so that $E^{\otimes n} a=0$, then $\left\langle E^{\otimes n}, E^{\otimes n}\right\rangle a=\left\langle E^{\otimes n}, E^{\otimes n} a\right\rangle=$ 0 . Hence, if $E^{\otimes n}$ is full, then $\operatorname{ann}\left(E^{\otimes n}\right)=0$.) Theorem 3.2 then implies that the $C^{*}$ envelope of $\mathcal{T}_{+}(E) / J_{n}$ is $K(P \mathcal{F}(E))$.

For the induction, assume that $E^{\otimes(n-1)}$ is essential and full. Then

$$
\begin{aligned}
\overline{\left\langle E^{\otimes n}, E^{\otimes n}\right\rangle} & =\overline{\left\langle E \otimes E^{\otimes(n-1)}, E \otimes E^{\otimes(n-1)}\right\rangle} \\
& =\overline{\left\langle E^{\otimes(n-1)}, \varphi(\langle E, E\rangle) E^{\otimes(n-1)}\right\rangle} \\
& =\overline{\left\langle E^{\otimes(n-1)}, \varphi(A) E^{\otimes(n-1)}\right\rangle} \\
& =\overline{\left\langle E^{\otimes(n-1)}, E^{\otimes(n-1)}\right\rangle} \\
& =A .
\end{aligned}
$$

Hence, $E^{\otimes n}$ is full. (In this string of equations, we used the fullness of $E$ and the fullness and essentiality of $E^{\otimes(n-1)}$.) Since $\varphi(A) E^{\otimes n}=(\varphi(A) E) \otimes E^{\otimes(n-1)}=E^{\otimes n}$, it is evident that $E^{\otimes n}$ is essential.

Example 4.1, below, shows that if the intersection of annihilators is non-zero, then the $C^{*}$-envelope of $\mathcal{T}_{+}(E) / \mathfrak{J}$ can be a proper quotient of $K(P \mathcal{F}(E)$ ).

\section{Quivers}

In this section we present a brief discussion of quivers and their algebras for the purposes of giving some examples and of putting the preceding results into perspective.

A quiver is simply a finite directed graph $(\mathcal{Q}, \mathcal{V}, r, s)$, where $r$ and $s$ are functions from $\mathcal{Q}$, the space of edges or arrows, to $\mathcal{V}$, the space of vertices. The maps $r$ and $s$ are called the range and source maps of the quiver, respectively, and one says that $\alpha \in \mathcal{Q}$ is an arrow from $s(\alpha)$ to $r(\alpha)$. Quivers are implicit in [6] and appeared sporadically in the ring-theory literature subsequently until 1972, when they were used with spectacular success by Gabriel. Since then, quivers have played an essential role in ring theory. For 
an encyclopaedic survey of their role in the representation theory of algebras see [5]. Given $(\mathcal{Q}, \mathcal{V}, r, s)$, let $A$ be the finite-dimensional $C^{*}$-algebra consisting of all $\mathbb{C}$-valued functions on $\mathcal{V}$ and let $E_{\mathcal{Q}}$ be the space of all $\mathbb{C}$-valued functions on $\mathcal{Q}$. Then $E_{\mathcal{Q}}$ has the structure of a $C^{*}$-correspondence over $A$. The right and left actions of $A$ on $E_{\mathcal{Q}}$ are given by

$$
\begin{aligned}
\xi \cdot f(\alpha) & :=\xi(\alpha) f(s(\alpha)) \\
\varphi(f) \xi(\alpha) & :=f(r(\alpha)) \xi(\alpha)
\end{aligned}
$$

$\xi \in E_{\mathcal{Q}}, f \in A, \alpha \in \mathcal{Q}$. The $A$-valued inner product on $E_{\mathcal{Q}}$ is given by the formula: $\langle\xi, \eta\rangle(v)=\sum_{s(\alpha)=v} \overline{\xi(\alpha)} \eta(\alpha)$. It is then easy to see that $E_{\mathcal{Q}}^{\otimes n}$ is just the space of functions on the fibred product $\mathcal{Q}^{(n)}=\left\{\left(\alpha_{n}, \alpha_{n-1}, \ldots, \alpha_{1}\right) \mid s\left(\alpha_{i+1}\right)=r\left(\alpha_{i}\right)\right\}$ which, in turn, is called the space of paths of length $n$, a tuple being a path. Each $\mathcal{Q}^{(n)}$ has an obvious pair of maps to $\mathcal{V}$, which we also denote by $r$ and $s$, and the correspondence structure on $E_{\mathcal{Q}}^{\otimes n}$ is given in terms of them: $r\left(\alpha_{n}, \alpha_{n-1}, \ldots, \alpha_{1}\right)=r\left(\alpha_{n}\right)$ and $s\left(\alpha_{n}, \alpha_{n-1}, \ldots, \alpha_{1}\right)=s\left(\alpha_{1}\right)$. The disjoint union $\mathcal{P}=\coprod_{n=0}^{\infty} \mathcal{Q}^{(n)}$, where $\mathcal{Q}^{(0)}:=\mathcal{V}$ is a small category called the path category of the quiver. The objects are just the vertices $\mathcal{V}$, and the morphisms from $v_{1} \in \mathcal{V}$ to $v_{2} \in \mathcal{V}$ are just the paths with source $v_{1}$ and range $v_{2}$; composition of paths is just concatenation. The Fock space $\mathcal{F}\left(E_{\mathcal{Q}}\right)$ may be realized as a completion of the space of all finitely supported functions on $\mathcal{P}$ endowed with the $A$-valued inner product: $\langle\xi, \eta\rangle(v)=\sum_{s(\underline{\alpha})=v} \overline{\xi(\underline{\alpha})} \eta(\underline{\alpha})$, where now the $\underline{\alpha}$ come from $\mathcal{P}$. The path algebra of $\mathcal{Q}$, $\mathbb{C} \mathcal{P}$, is simply the space of all finitely supported functions on $\mathcal{P}$, where the product is given by the formula

$$
f * g(\underline{\gamma})=\sum_{\underline{\alpha} \underline{\beta}=\underline{\gamma}} f(\underline{\alpha}) g(\underline{\beta}) .
$$

The map $\lambda$ defined by the formula $\lambda(f) g=f * g, f \in \mathbb{C} \mathcal{P}$, but with $g$ viewed as an element of $\mathcal{F}\left(E_{\mathcal{Q}}\right)$, is a representation of $\mathbb{C} \mathcal{P}$ with values in $\mathcal{L}\left(\mathcal{F}\left(E_{\mathcal{Q}}\right)\right)$. In fact, for functions $\xi$ supported on $\mathcal{Q} \subseteq \mathcal{P}, T_{\xi}=\lambda(\xi)$, and $\mathcal{T}_{+}\left(E_{\mathcal{Q}}\right)$ is the closure of $\lambda(\mathbb{C} \mathcal{P})$ in $\mathcal{L}\left(\mathcal{F}\left(E_{\mathcal{Q}}\right)\right)$. One of the principal results of [9] is that if the maps $r$ and $s$ in the quiver are surjective, then the $C^{*}$-envelope of $\mathcal{T}_{+}\left(E_{\mathcal{Q}}\right)$ in this case is a Cuntz-Krieger algebra, $\mathcal{O}_{A}$, where the incidence matrix $A$ is constructed in a natural way from $\mathcal{Q}$.

A relation on the quiver $(\mathcal{Q}, \mathcal{V}, r, s)$ is a finite $\mathbb{C}$-linear combination paths $c_{1} \underline{\gamma}_{1}+c_{2} \underline{\gamma}_{2}+$ $\cdots+c_{k} \underline{\gamma}_{k}, \underline{\gamma}_{i} \in \mathcal{P}$ (possibly of different lengths), such that the paths in the combination have the same source and the same range. An ideal $\mathfrak{I}$ in $\mathbb{C P}$ is called admissible in case it is generated by a finite number of relations and is nested between $J_{1}$ and $J_{n}$, for some finite $n$, where $J_{k}$ consists of all functions in $\mathbb{C P}$ that are supported on $\coprod_{j \geqslant k} \mathcal{Q}^{(j)}$. For our purposes, one of the basic theorems about finite-dimensional algebras over $\mathbb{C}$ is that every such algebra is (algebraically) Morita equivalent to a quotient $\mathbb{C P} / \mathfrak{I}$, where $\mathbb{C} \mathcal{P}$ is the path algebra of a suitable quiver and $\mathfrak{I} \subseteq \mathbb{C P}$ is an admissible ideal. Since we are dealing with finite-dimensional objects, this fact may also be stated: every finitedimensional operator algebra is $c b$-Morita equivalent, in the sense of $[2]$, to $\mathcal{T}_{+}\left(E_{\mathcal{Q}}\right) / \overline{\mathfrak{I}}$ for a suitable quiver $\mathcal{Q}$ and admissible ideal $\mathfrak{I} \subseteq \mathbb{C P}$, where $\overline{\mathfrak{I}}$ is the closure of $\mathfrak{I}$ in 
$\mathcal{T}_{+}\left(E_{\mathcal{Q}}\right)$. Thus, quotients of tensor algebras of $C^{*}$-correspondences contain models for all finite-dimensional operator algebras.

Observe that (the closures of) admissible ideals satisfy hypotheses (H1) and (H3) above. They need not satisfy hypothesis (H2), however. An admissible ideal will satisfy this hypothesis provided it is spanned by the homogeneous relations it contains. (A relation is homogeneous if the paths entering into it all have the same length.) Assuming this, then since hypothesis $(\mathrm{H} 4)$ is automatic, we see that the $C^{*}$-envelope of $\mathcal{T}_{+}\left(E_{\mathcal{Q}}\right) / \overline{\mathfrak{I}}$ is calculated by our theorem if the intersection of the annihilators in hypothesis (H5) vanishes. This can, in principle, be decided for each prescribed quiver.

Better still, even if the annihilation condition is not satisfied, one can still calculate the Shilov boundary ideal, and, ultimately, the $C^{*}$-envelope of the quotient $\mathcal{T}_{+}\left(E_{\mathcal{Q}}\right) / \overline{\mathfrak{I}}$, assuming homogeneity of $\mathfrak{I}$. This is quite complicated to articulate in general, but it is illustrated by the following example, which also shows that conditions on the annihilators are necessary in order to calculate $C^{*}$-envelopes of the quotients we are contemplating.

Example 4.1. Continuing with the notation just established, observe that every ideal in $A$ is given by a projection in $A$. The annihilator of $E_{\mathcal{Q}}$, ann $\left(E_{\mathcal{Q}}\right)$, consists of those functions on $\mathcal{V}$ that are supported in the complement of the range of $s$. Assume this complement is non-empty and write $\operatorname{ann}\left(E_{\mathcal{Q}}\right)=F A$, where $F$ is its characteristic function. Also, assume that $k\left(E_{\mathcal{Q}}\right)$ is proper and write $k\left(E_{\mathcal{Q}}\right)=G A$, where $G$ is the characteristic function of the complement of $r(\mathcal{Q})$. For our ideal $\mathfrak{I}$ in the tensor algebra $\mathcal{T}_{+}\left(E_{\mathcal{Q}}\right)$, simply take $\mathfrak{I}=J_{2}$. Hypotheses (H1)-(H3) are satisfied immediately. Hypothesis (H4) is satisfied because $E_{\mathcal{Q}}$ is finite dimensional. What is at issue is hypothesis (H5).

In our setting, $P=P_{2}$ and $K\left(P \mathcal{F}\left(E_{\mathcal{Q}}\right)\right)=K\left(A \oplus E_{\mathcal{Q}}\right)$. Hypothesis (H5) becomes $F G^{\perp}=0$. So, in general, the $C^{*}$-envelope of $\mathcal{T}_{+}\left(E_{\mathcal{Q}}\right) / \mathfrak{I}$ might differ from $K\left(A \oplus E_{\mathcal{Q}}\right)$. In fact, it is our aim here to show that the $C^{*}$-envelope is always $K\left(A\left(1-F G^{\perp}\right) \oplus E_{\mathcal{Q}}\right)$. For this we set $\mathfrak{I}_{1}=A F G^{\perp}(\subseteq A)$ and show that $K\left(P \mathcal{F}\left(E_{\mathcal{Q}}\right) \mathfrak{I}_{1}\right)$ is the maximal boundary ideal (i.e. the Shilov ideal) in $K\left(P \mathcal{F}\left(E_{\mathcal{Q}}\right)\right.$ ) for $P \mathcal{T}_{+}\left(E_{\mathcal{Q}}\right) P$.

First note that $\operatorname{ann}\left(E_{\mathcal{Q}}\right) \cap \operatorname{ann}\left(k\left(E_{\mathcal{Q}}\right)\right)=A F G^{\perp}\left(=\mathfrak{I}_{1}\right)$ and it follows from the proof of Theorem 3.2 that for every non-zero ideal $\mathfrak{I}_{0} \subseteq A$ with $\mathfrak{I}_{0} \nsubseteq \mathfrak{I}_{1}, K\left(P \mathcal{F}\left(E_{\mathcal{Q}}\right) \mathfrak{I}_{0}\right)$ is not a boundary ideal. Hence, it suffices to show that $K\left(P \mathcal{F}\left(E_{\mathcal{Q}}\right) \mathfrak{I}_{1}\right)$ is a boundary ideal. Let $Q$ be the projection onto $A\left(I-F G^{\perp}\right) \oplus E_{\mathcal{Q}}$. Note that $K\left(P \mathcal{F}\left(E_{\mathcal{Q}}\right) \mathfrak{I}_{1}\right)=K\left(\left(A \oplus E_{\mathcal{Q}}\right) \mathfrak{I}_{1}\right)=$ $K\left(\left(A \oplus E_{\mathcal{Q}}\right) A F G^{\perp}\right)=K\left(A F G^{\perp}\right)$. Hence, the quotient $K\left(P \mathcal{F}\left(E_{\mathcal{Q}}\right)\right) / K\left(P \mathcal{F}\left(E_{\mathcal{Q}}\right) \mathfrak{I}_{1}\right)$ can be identified with $K\left(Q \mathcal{F}\left(E_{\mathcal{Q}}\right)\right.$ ), and the quotient map becomes the compression by $Q$, using this identification.

It suffices to prove that the map $P T P \mapsto Q T Q, T \in \mathcal{T}_{+}\left(E_{\mathcal{Q}}\right)$, is a complete isometry. Since this map is multiplicative on $K\left(A \oplus E_{\mathcal{Q}}\right)$, it follows that

$$
P T P=Q T Q+(P-Q) T(P-Q), \quad T \in \mathcal{T}_{+}\left(E_{\mathcal{Q}}\right)
$$

and it is enough to show that the map

$$
Q T Q \longmapsto(P-Q) T(P-Q), \quad T \in \mathcal{T}_{+}\left(E_{\mathcal{Q}}\right),
$$

is completely contractive. 
Note that $P-Q$ is the projection onto $A F G^{\perp}$ and that $A F G^{\perp} \otimes_{A} E_{\mathcal{Q}}=A F \otimes_{A} E_{\mathcal{Q}} \cong$ $\varphi(A F) E_{\mathcal{Q}}$. If we write $R$ for the projection onto $\varphi(A F) E_{\mathcal{Q}}$ we can, for $T \in \mathcal{T}_{+}\left(E_{\mathcal{Q}}\right)$; identify $(P-Q) T(P-Q) \otimes I_{E_{\mathcal{Q}}}$ with $R T R$ and, thus, the map

$$
(P-Q) T(P-Q) \longmapsto R T R, \quad T \in \mathcal{T}_{+}\left(E_{\mathcal{Q}}\right),
$$

is a complete isometry. As $R \leqslant Q$, the map

$$
Q T Q \longmapsto(P-Q) T(P-Q), \quad T \in \mathcal{T}_{+}\left(E_{\mathcal{Q}}\right),
$$

is completely contractive. This completes the proof.

The heart of any study of an algebra of the form $\mathcal{T}_{+}(E) / \mathfrak{I}$ (indeed of any abstractly presented operator algebra in general) is to find an effective means of realizing it completely isometrically in some $C^{*}$-algebra. That is the role of hypotheses (H1)-(H3) in this paper. One might ask, however, if perhaps the following straightforward, naive approach might work. Simply identify $\mathfrak{I}$ with $\left\{\xi \in \mathcal{F}(E) \mid T_{\xi} \in \mathfrak{I}\right\}$, form its closure in $\mathcal{F}(E)$, and compress to its orthogonal complement, assuming the closure is complemented. This suggestion was made in $[8$, p. 349]. We conclude with an example that shows this approach to be ineffective in general, and, therefore, that some hypotheses are needed to imbed $\mathcal{T}_{+}(E) / \mathfrak{I}$ nicely in a $C^{*}$-algebra. We chose the ones that we did because of their connection with the finite-dimensional, purely algebraic situation.

Example 4.2. Let $(\mathcal{Q}, \mathcal{V}, r, s)$ be the quiver with but one vertex $v$ and two arrows $\alpha_{1}$ and $\alpha_{2}$ from $v$ to $v$. Then $A=\mathbb{C}$ and $E_{\mathcal{Q}}$ is two-dimensional Hilbert space, but with the inner product conjugate linear in the second variable. Then $\mathcal{T}_{+}\left(E_{\mathcal{Q}}\right)$ is the noncommutative disc algebra studied by Popescu in [13]. The path space $\mathcal{P}$ is simply the free monoid on the two generators, $\alpha_{1}$ and $\alpha_{2}$, with $v$ serving as the identity. The Fock space correspondence $\mathcal{F}\left(E_{\mathcal{Q}}\right)$ is simply $\ell^{2}(\mathcal{P})$ and has an orthonormal basis consisting of the characteristic functions of the words in $\alpha_{1}$ and $\alpha_{2}$ and the function $1_{\{v\}}$.

The map that sends $\alpha_{1}$ to the matrix unit $e_{12}$ in $M_{2}(\mathbb{C})$ and sends $\alpha_{2}$ to $e_{21}$ extends to a completely contractive representation $\rho$ of $\mathcal{T}_{+}\left(E_{\mathcal{Q}}\right)$ onto $M_{2}(\mathbb{C})$. (The fact that $\rho$ is completely contractive follows from the fact that $\rho\left(\alpha_{1}\right) \rho\left(\alpha_{1}\right)^{*}+\rho\left(\alpha_{2}\right) \rho\left(\alpha_{2}\right)^{*}=$ $e_{12} e_{12}^{*}+e_{21} e_{21}^{*}=1$ and Popescu's dilation theorem [12] (see also [9, Theorem 3.9 and Corollary 3.15]).) Let $\mathfrak{I}$ be the kernel of this representation. We aim to show that $\mathfrak{I}$ is dense in $\ell^{2}(\mathcal{P})$. For $\xi \in \ell^{2}(\mathcal{P})$, we write $\hat{\xi}(w)$ for the Fourier coefficient determined by $w \in \mathcal{P}$, i.e. $\hat{\xi}(w)=\left(1_{\{w\}}, \xi\right)$, where $1_{\{w\}}$ is the characteristic function of $\{w\}$. Suppose that $\xi$ is orthogonal to $\mathfrak{I}$ and observe that if $w$ is any non-empty word ending, say, in $\alpha_{1}$, then $1_{\{w\}}-1_{\left\{w\left(\alpha_{2} \alpha_{1}\right)^{n}\right\}}$ lies in $\mathfrak{I}$ for all $n \geqslant 0$. Likewise, if $w$ ends in $\alpha_{2}$, then $1_{\{w\}}-$ $1_{\left\{w\left(\alpha_{1} \alpha_{2}\right)^{n}\right\}} \in \mathfrak{I}$ for all $n \geqslant 0$. Since $\xi \perp \mathfrak{I}$, we have $\hat{\xi}(w)=\hat{\xi}\left(w\left(\alpha_{2} \alpha_{1}\right)^{n}\right) \rightarrow 0$, if $w$ ends in $\alpha_{1}$, and this implies that $\hat{\xi}(w)=0$. Likewise, $\hat{\xi}(w)=0$, if $w$ ends in $\alpha_{2}$. That leaves us to show $\hat{\xi}(v)=0$. However, since $\rho\left(1_{v}\right)=1=e_{12} e_{12}^{*}+e_{21} e_{21}^{*}=\rho\left(1_{\left\{\alpha_{1} \alpha_{2}\right\}}\right)+\rho\left(1_{\left\{\alpha_{2} \alpha_{1}\right\}}\right)$, we see that $1_{v}-\left(1_{\left\{\alpha_{1} \alpha_{2}\left(\alpha_{1} \alpha_{2}\right)^{n}\right\}}+1_{\left\{\alpha_{2} \alpha_{1}\left(\alpha_{2} \alpha_{1}\right)^{m}\right\}}\right) \in \mathcal{J}$ for all $m, n \geqslant 0$; and again we conclude that $\hat{\xi}(v)=0$. 
Acknowledgements. P.S.M. gratefully acknowledges support by grants from the US-Israel Binational Science Foundation and the US National Science Foundation. B.S. gratefully acknowledges support by grants from the US-Israel Binational Science Foundation and by the Fund for the Promotion of Research at the Technion.

\section{References}

1. W. ARVeson, Subalgebras of $C^{*}$-algebras, Acta Math. 123 (1969), 141-224.

2. D. Blecher, P. Muhly and V. Paulsen, Categories of operator modules (Morita equivalence and projective modules), Mem. Am. Math. Soc. 143, no. 681 (2000).

3. K. Davidson, Nest algebras, Pitman Research Notes in Mathematics Series, vol. 191 (Longman Scientific and Technical, Essex, 1988).

4. C. Davis, W. Kahan and W. Weinberger, Norm preserving dilations and their applications to optimal error bounds, SIAM J. Numer. Analysis 19 (1982), 445-469.

5. P. Gabriel AND A. RoIter, Representations of finite-dimensional algebras, in Algebra VIII, Encyclopaedia of Mathematical Sciences (Springer, 1992).

6. G. HoCHSCHILD, On the structure of algebras with nonzero radical, Bull. Am. Math. Soc. 53 (1947), 369-377.

7. E. C. LANCE, Hilbert $C^{*}$-modules-a toolkit for operator algebraists, London Mathematical Society Lecture Notes (Cambridge University Press, 1995).

8. P. S. MUHLY, A finite dimensional introduction to operator algebra, in Operator algebras and applications (ed. A. Katavolos), pp. 313-354, NATO Adv. Sci. Inst. C, Math. Phys. Sci., vol. 495 (Kluwer, Dordrecht, 1997).

9. P. Muhly AND B. Solel, Tensor algebras over $C^{*}$-correspondences (representations, dilations, and $C^{*}$-envelopes), J. Funct. Analysis 158 (1998), 389-457.

10. P. Muhly and B. Solel, On the Morita equivalence of tensor algebras, Proc. Lond. Math. Soc. (In the press.)

11. M. PIMSNER, A class of $C^{*}$-algebras generalizing both Cuntz-Krieger algebras and crossed products by $\mathbb{Z}$, in Free probability theory (ed. D. Voiculescu), Fields Institute Communications, vol. 12, pp. 189-212 (American Mathematical Society, Providence, RI, 1997).

12. G. POPESCU, Isometric dilations for infinite sequences of noncommuting operators, Trans. Am. Math. Soc. 316 (1989), 523-536.

13. G. POPESCU, Noncommuting disc algebras and their representations, Proc. Am. Math. Soc. 124 (1996), 2137-2148.

14. M. RiEFFEL, Induced representations of $C^{*}$-algebras, Adv. Math. 13 (1974), 176-257.

15. B. SOLEL, Operator algebras over $C^{*}$-correspondences, in Operator algebras and applications (ed. A. Katavolos), pp. 429-448, NATO Adv. Sci. Inst. C, Math. Phys. Sci., vol. 495 (Kluwer, Dordrecht, 1997). 